\title{
Protective effects of ethanolic extract of Alhagi maurorum roots on renal failure induced by acetaminophen in mice
}

\begin{abstract}
The protective actions of ethanol Alhagi maurorum (AM) root ethanol extract on acetaminophen-induced oxidative stress and renal toxicity in mice was evaluated. Forty male SWR strain albino mice aged 8 weeks were grouped into five groups. G1 $(n=5)$ : as control. G2 $(\mathrm{n}=5)$ : administered orally a single dose of acetaminophen $(2000 \mathrm{mg} / \mathrm{kg})$. G3 $(\mathrm{n}=10)$ administrated orally $200 \mathrm{mg} / \mathrm{kg}$ of roots ethanol extract for one week then acetaminophen as G2 at $8^{\text {th }}$ day and; G4 (n=10) administrated orally $400 \mathrm{mg} / \mathrm{kg}$ of roots ethanol extract for one week then acetaminophen as G2 at $8^{\text {th }}$ day; G5 $(n=10)$ administrated orally $600 \mathrm{mg} / \mathrm{kg}$ of roots ethanol extract for one week then acetaminophen as G2 at $8^{\text {th }}$ day. At end of experiments, the mice were killed under anesthesia and blood samples were gathered to preform complete blood test (CBC), serum levels of urea and creatinine and oxidative stress biomarkers as superoxide dismutase (SOD), glutathione (GSH), and catalase (CAT) using available Elisa mice kits. Kidneys were removed and histologically examined. Acetaminophen intake significantly elevated WBCs, neutrophils, monocytes, urea and creatinine levels and significantly decreased RBCs, hemoglobin, hematocrit, GSH, SOD and CAT $(P<0.05)$. Treatment with Alhagi maurorum roots extract especially high dose $(600 \mathrm{mg} / \mathrm{kg})$ resulted in decreased in WBCs, neutrophils, monocytes, urea and creatinine levels and significantly increased RBCs, hemoglobin, hematocrit, GSH, SOD and CATversusacetaminophen group. Alhagi maurorum root extract treatment similarly decreased renal histological alteration induced by acetaminophen. This study can be utilized as prove of reading that Alhagi maurorum ethanol root extract especially high dose might be administered to prevent renal destruction induced by acetaminophen due to its antioxidant activity.
\end{abstract}

Keywords: acetaminophen, Alhagi maurorum, mice, renal failure, toxicity
Volume 8 Issue I - 202 I

\author{
Seham I AL-Nafea, Mohammed O Aljahdali \\ Biological Sciences Department, King Abdulaziz University, Saudi \\ Arabia
}

Correspondence: Mohammed O Aljahdali, Biological Sciences Department, King Abdulaziz University, Faculty of Science, P.O Box 80203 Jeddah 21589, Saudi Arabia,

Emailmoaljahdal@kau.edu.sa

Received: December 28, 2020 | Published: February 17, 202
Abbreviations: SOD, superoxide dismutase; CAT, catalas; AM, Alhagi maurorum

\section{Introduction}

Medical plants play important role in all the world's population life since the ancient times in folk medicine, to relieve pain and treat diseases. They widely used by patients due to for their availability, effectiveness, fewer side effects, and low cost. ${ }^{1}$ Despite the benefits of medicinal plants, some of them have harmful side effects due to use of overdose or for long period or because it is toxic or other factors. ${ }^{2}$ Medicinal plants are future resource of new drugs and pharmaceuticals. Therefore, WHO and others of international organizations have urged using traditional medicine and medical herbs for diseases treatments and preventions. ${ }^{3}$

Alhagi maurorum (AM) is one of these plants; it has been widely used for several medicinal purposes. ${ }^{3}$ Alhagi maurorum is a perennial plant from Fabacea family and Fabales order. It grows abundantly in Saudi Arabia, Middle East and other areas of the world. It has many common names like Camel Thorn, Persian manna-plant, or Caspian manna. ${ }^{4}$ The plant is used in traditional medicine as a laxative, diaphoretic, purgative, diuretic and expectorant. Its flower extracts are used as a medicine to treat of piles, warts, and migraine. Its leaves extracts used as oil for treat of rheumatism. Its roots extracts used to remove kidney stones by relaxation of urete. ${ }^{5}$ Alhagi maurorum is rich in flavonoid, phenolic compounds, glycosides, anthraquinone, saponins, steroids, tannins, and alkaloids. These compounds are inhibitors of lipid peroxidation. Flavonoids are the secondary metabolites that have role as antioxidant, anti-allergic, antiinflammatory, antibacterial, antiviral and antifungal effects. ${ }^{6,7}$

Renal failure is complete or partial damage of kidney functions that may be chronic and acute. ${ }^{8}$ The life of patients with renal failure depends on dialysis or kidney transplant which are costly. Li et al., reported that ethanolextract of Chinese chive water in a concentration of $200 \mathrm{mg} / \mathrm{kg} /$ day given to adenine-induced chronic renal failure (CRF) mice model led to significant decrease of renal pathological damage to $48.1 \%$, and decrease values of serum creatinine and blood urea nitrogen to $87.7 \%$ and $83.9 \%$, respectively. Therefore, it is urgently need to provide a nonconventional, low-cost medicine for renal failure patients.

Pharmacological activity of ethanolextract for roots of Alhagi maurorum showed the following: 1) decreased the body temperature in mice in doses of $1 \mathrm{~g} / \mathrm{kg}^{2}$ ) relaxation of a skeletal muscle of frog 3) reduced heart rate by $22.5 \%$ at dose of $1 \mathrm{~g} / \mathrm{kg}$ in rats 4) relaxations of ureter in guinea-pig. ${ }^{5}$ Ethanol extract of aerial parts of Alhagi maurorum had anti-inflammatory, anti-nociceptive and antipyretic activity in lab animals at doses of 500 and $1000 \mathrm{mg} / \mathrm{kg} .{ }^{10}$ Ethanol extract of Alhagi maurorum in a dose of $660 \mathrm{mg} / \mathrm{kg}$ of body weight for mice had a big hepatoprotective activity against acetaminophen and carbon tetrachloride side effects. ${ }^{6}$ Ethanol extract of Alhagi maurorum has anti-inflammatory and anti-ulcer activity at the dose daily $100 \mathrm{mg} / \mathrm{kg}$ of body weight for rat. ${ }^{11}$ Methanol extract of aerial parts of Alhagi maurorum has antidiarrheal activity at 200 and 400 $\mathrm{mg} / \mathrm{kg} .^{3}$ The objective of this experimental study was to investigate the effect of oral intake of different concentrations $(200,400,600$ 
$\mathrm{mg} / \mathrm{kg}$ ) for one week of ethanol extract of Alhagi maurorum roots on hematological, kidney function parameters and oxidative stress markers of acetaminophen induced kidney injury mice model.

\section{Materials and methods}

\section{Experimental animals}

All experimental steps with mice were approved by ethical guidelines of experimental animal care and use committee of King Abdulaziz University (KAU Reference \# 572-19). Fortyalbino mice of SWR strain aged 8 weeks old and weighting 23-25 gramsutilized in this experimental study. Mice wereget from animal house unit of King Fahad Medical Research Centre (KFMRC), KAU, Jeddah, Saudi Arabia. Mice were acclimatized to laboratory status for 1 week before to beginning of experiments. Animals wereput in plastic cages and maintained in laboratory status of room temperature $\left(20 \pm 1 \mathrm{C}^{\circ}\right)$, light: dark cycle (12:12h) and humidity (65\%). Mice fed ad libitum with rodentfood and had free availability to tap water.

\section{Experimental design}

Animals sorted into five groups as follow G1 $(n=5)$ : control group received no treated; G2 $(\mathrm{n}=5)$ : received $2000 \mathrm{mg} / \mathrm{kg}$ of acetaminophen once orally to induced to induce kidney injury, ${ }^{12}$ G3 ( $\mathrm{n}=10)$ administrated orally $200 \mathrm{mg} / \mathrm{kg}$ of ethanol extract of Alhagi maurorum roots (AM) for one week then acetaminophen at $8^{\text {th }}$ day; G3 (n=10) administrated orally $400 \mathrm{mg} / \mathrm{kg}$ of ethanol extract of Alhagi maurorum roots for one week then acetaminophen at $8^{\text {th }}$ day; G5 (n=10) administrated orally $600 \mathrm{mg} / \mathrm{kg}$ of ethanol extract of Alhagi maurorum roots for one weekthen acetaminophen at $8^{\text {th }}$ day.

\section{Plant collections and preparation of aqueous extract}

Fresh herb of Alhagi maurorum was gathered from the desert between Qassim and Riyadh area. The plant was identified by comparison with authentic sample in the herbarium of Biological Sciences Department, Faculty of Science, King Abdulaziz University. Plants were washed with tap water and left for drying in air. Approximately, $300 \mathrm{~g}$ of plant rootwas dried and root powders were extracted by continuous exhalation with ethanol $(500 \mathrm{ml})$. Then fractions evaporated to dryness utilizing rota vapor (R-100 Rotary Evaporator Thomson Scientific). ${ }^{6}$

\section{Hematological data}

At experimental end, blood was gathered from retro-orbital veins into plain tube. Blood was centrifuged 11,000 cycles per minute for $15 \mathrm{~min}$. Sera was obtained aliquot and stored at $-20^{\circ} \mathrm{C}$ till used. Complete blood count (CBC) measured by using manual differential method to determine number of each cell types (WBC, RBC, HB, PLT) according to A Lab 1315 Hematology. Mice ELISA kits were used for estimation of serum levels of urea, creatinine, glutathione (GSH), superoxide dismutase (SOD) and catalase (CAT) according to the manufacture protocol.

\section{Histological examination}

At end of the experiment, rats were killed by cervical dislocation after inhalation of light diethyl ether anesthesia. Abdomen was dissected and kidneys were excised, washed with water and dried. Formalin $(10 \%)$ was utilized to fix renal tissues that were altered many times for good tissues processing. The tissues were treated with graded xylene and ethanol. The processed tissues were put into paraffin pieces to inhibit air entry. The paraffin blocks-containing tissues were cut into slices of approximately five $\mu \mathrm{m}$ thickness utilized a rotary microtome machine. After slicing, tissues were put on glass slides and stained with hematoxylin and eosin (H\&E) based on regular methods. Stained tissue sections were examined for any structural alterations, and their photographs were taken under a light microscope.

\section{Data analysis}

Data were expresses as mean \pm standard deviation and analyzed by IBM SPSS Statistics for Windows, version 23 (IBM SPSS, IBM Corp., Armonk, N.Y., USA). Shapiro - Wilk test was used to evaluate normal data distribution. OneWay ANOVA test then least significant difference (LSD), assuming groups' equal variance, utilized to calculate significance. P-values of $<0.05$ considered statistically significant.

\section{Study limitations}

Collecting Alhagi maurorum from the field was difficult due to the rare availability of this herb. The appropriate dose of acetaminophen was tough to determine with water insolubility of acetaminophen. Finally, controlling the mortality rate during renal failure induction at the lab was a big challenge to us.

\section{Results}

WBCs count were significantly increased in G2, G3 and G4 groups versus $\mathrm{G} 1(\mathrm{P}=0.001, \mathrm{P}=0.003$ and $\mathrm{P}=0.026)$ but significantly increased in G5 after treatment with AM in high dose $600 \mathrm{mg} / \mathrm{kg}$ versus $\mathrm{G} 2, \mathrm{G} 3$ and $\mathrm{G} 4(\mathrm{P}=0.001, \mathrm{P}=0.004$ and $\mathrm{P}=0.039)$. Neutrophil and basophil (\%) were significantly increased in G2 (acetaminophen treated group) versus $\mathrm{G} 1$ (control) $(\mathrm{P}<0.0001$ and $\mathrm{P}=0.017)$, and AM treated groups $\mathrm{G} 3(\mathrm{P}=0.008$ and $\mathrm{P}=0.038), \mathrm{G} 4(\mathrm{P}=0.002$ and $\mathrm{P}=0.017)$ and $\mathrm{G} 5(\mathrm{P}=0.001$ and $\mathrm{P}=0.017)$. Also, monocytes $(\%)$ were significantly increased in G2 (acetaminophen treated group) versus $\mathrm{G} 1$ (control), and AM treated groups $\mathrm{G} 4$ and $\mathrm{G} 5(\mathrm{P}=0.009, \mathrm{P}=0.027$, and $\mathrm{P}=0.005$, respectively) and in $\mathrm{G} 3$ versus $\mathrm{G} 5(\mathrm{P}=0.047)$. RBCs count was significantly decreased in G2 (acetaminophen treated group) versus $\mathrm{G} 1$ (control), and AM treated group G5 ( $\mathrm{P}=0.011$ and $\mathrm{P}=0.013)$. Hemoglobin values were significantly decreased in in $\mathrm{G} 2$ (acetaminophen treated group) versus G1 (control), and AM treated groups G3, G4 and G5 ( $<<0.0001$ for all). After treatment with AM, hemoglobin levels increased but were still significantly lower than control group in $\mathrm{G} 3$ and $\mathrm{G} 4(\mathrm{P}=0.004$ and $\mathrm{P}=0.019)$. Hemoglobin level was significantly increased in G5 versus G3 $(\mathrm{P}=0.011)$. Hematocrit levels were significantly decreased in in G2 (acetaminophen treated group) versus G1 (control), and AM treated groups G3, G4 and G5 $(\mathrm{P}<0.0001$ for all). After treatment with AM, HCT levels increased but were still significantly lower than control group in $\mathrm{G} 3(\mathrm{P}=0.033)$. HCT levels were significantly increased in G4 and G5 versus G3 $(\mathrm{P}=0.010$ and $\mathrm{P}=0.025)$ (Table 1).

Serum urea values were significantly increased in G2, G3, G4 versus control $(\mathrm{P}<0.0001, \mathrm{P}=0.001$ and $\mathrm{P}=0.034)$; but significantly decreased in G3, G4 and G5 versus G2 $(\mathrm{P}<0.0001$ for all) and in G5 versus G3 ( $\mathrm{P}=0.013)$ (Table 2 \& Figure 1$)$. GSH levels were significantly declinedin $\mathrm{G} 2, \mathrm{G} 3$, G4 versus control $(\mathrm{P}=0.005, \mathrm{P}=0.011$ and $\mathrm{P}=0.022)$; but significantly elevated in $\mathrm{G} 5$ versus $\mathrm{G} 2(\mathrm{P}=0.038)$. SOD levels were significantly decreased in G2, G3, G4 versus control $(\mathrm{P}<0.0001, \mathrm{P}<0.0001$ and $\mathrm{P}=0.005)$; but significantly elevated in $\mathrm{G} 3$, $\mathrm{G} 4$ and G5 versus $\mathrm{G} 2(\mathrm{P}<0.0001$ for all) and in G4 and G5 versus $\mathrm{G} 3(\mathrm{P}<0.0001$ for both) and in $\mathrm{G} 5$ versus $\mathrm{G} 4(\mathrm{P}=0.027)$. CAT levels were significantly decreased in G2, G3, G4 and G5 versus control $(\mathrm{P}<0.0001, \mathrm{P}<0.0001, \mathrm{P}=0.001$ and $\mathrm{P}=0.024)$; but significantly increased in $\mathrm{G} 5$ versus $\mathrm{G} 2$ and $\mathrm{G} 3(\mathrm{P}=0.002$ and $\mathrm{P}=0.023)$ (Table 3 \& Figure 2). 
Table I Comparison of complete blood count (CBC) between different studied groups

\begin{tabular}{|c|c|c|c|c|c|}
\hline Parameters & $\begin{array}{l}\text { GI } \\
\text { (Control) }\end{array}$ & $\begin{array}{l}\text { G2 } \\
\text { (acetaminophen) }\end{array}$ & $\begin{array}{l}\text { G3 } \\
\text { (AM } 200 \mathrm{mg} / \mathrm{kg})\end{array}$ & $\begin{array}{l}\text { G4 } \\
\text { (AM } 400 \mathrm{mg} / \mathrm{kg})\end{array}$ & $\begin{array}{l}\text { G5 } \\
(A M 600 \mathrm{mg} / \mathrm{kg})\end{array}$ \\
\hline WBCs $\left(x \mid 0^{3} / u l\right)$ & $3.90 \pm 0.95$ & $7.90 \pm 1.41$ & $7.30 \pm 1.40$ & $6.13 \pm 0.68$ & $4.10 \pm 0.44$ \\
\hline Significance & - & ${ }^{I} P=0.001$ & ${ }^{I} \mathrm{P}=0.003 ;{ }^{2} \mathrm{P}=0.500$ & $\begin{array}{l}{ }^{I} P=0.026 ;{ }^{2} P=0.066 \\
{ }^{3} P=0.203\end{array}$ & $\begin{array}{l}{ }^{1} P=0.820 ;{ }^{2} P=0.00 I ;{ }^{3} P=0.004 ; \\
{ }^{4} P=0.039\end{array}$ \\
\hline Neutrophil (\%) & $2.10 \pm 1.17$ & $6.77 \pm 1.20$ & $3.90 \pm 0.75$ & $3.17 \pm 1.42$ & $2.50 \pm 0.36$ \\
\hline Significance & - & 'P $<0.000$ I & ${ }^{I} \mathrm{P}=0.063 ;{ }^{2} \mathrm{P}=0.008$ & $\begin{array}{l}{ }^{\mathrm{P}} \mathrm{P}=0.243 ;{ }^{2} \mathrm{P}=0.002 \\
{ }^{3} \mathrm{P}=0.413\end{array}$ & $\begin{array}{l}{ }^{1} P=0.652 ;{ }^{2} P=0.00 I ;{ }^{3} P=0.134 ; \\
{ }^{4} P=0.456\end{array}$ \\
\hline Lymphocyte (\%) & $91.77 \pm 0.58$ & $96.87 \pm 1.46$ & $94.97 \pm 2.95$ & $83.80 \pm 22.61$ & $86.77 \pm 18.53$ \\
\hline Significance & - & ${ }^{\prime} P=0.645$ & $\mathrm{I} P=0.772 ;{ }^{2} \mathrm{P}=0.863$ & $\begin{array}{l}{ }^{1} P=0.475 ;{ }^{2} P=0.252 \\
{ }^{3} P=0.323\end{array}$ & $\begin{array}{l}{ }^{1} \mathrm{P}=0.652 ;{ }^{2} \mathrm{P}=0.369 ;{ }^{3} \mathrm{P}=0.463 ; \\
{ }^{4} \mathrm{P}=0.788\end{array}$ \\
\hline Monocytes (\%) & $0.40 \pm 0.10$ & $0.73 \pm 0.15$ & $0.60 \pm 0.10$ & $0.47 \pm 0.15$ & $0.37 \pm 0.12$ \\
\hline Significance & - & ${ }^{\prime} P=0.009$ & ${ }^{I} \mathrm{P}=0.082 ;{ }^{2} \mathrm{P}=0.226$ & $\begin{array}{l}{ }^{1} P=0.533 ;{ }^{2} P=0.027 \\
{ }^{3} P=0.226\end{array}$ & $\begin{array}{l}{ }^{I} P=0.754 ;{ }^{2} P=0.005 \\
{ }^{3} P=0.047 ;{ }^{4} P=0.356\end{array}$ \\
\hline Eosinophil (\%) & $0.47 \pm 0.06$ & $0.33 \pm 0.15$ & $0.30 \pm 0.10$ & $0.37 \pm 0.15$ & $0.30 \pm 0.10$ \\
\hline Significance & - & ${ }^{\prime} P=0.198$ & ${ }^{1} \mathrm{P}=0.1115 ;{ }^{2} \mathrm{P}=0.737$ & $\begin{array}{l}{ }^{\mathrm{P}} \mathrm{P}=0.325 ;{ }^{2} \mathrm{P}=0.737 \\
{ }^{3} \mathrm{P}=0.485\end{array}$ & $\begin{array}{l}{ }^{1} P=0.115 ;{ }^{2} P=0.737 ;{ }^{3} P=1.00 \\
{ }^{4} P=0.506\end{array}$ \\
\hline Basophil (\%) & $0.13 \pm 0.06$ & $0.33 \pm 0.15$ & $0.17 \pm 0.06$ & $0.13 \pm 0.06$ & $0.13 \pm 0.06$ \\
\hline Significance & - & ${ }^{I} P=0.017$ & ${ }^{I} \mathrm{P}=0.644 ;{ }^{2} \mathrm{P}=0.038$ & $\begin{array}{l}{ }^{1} \mathrm{P}=1.00 ;{ }^{2} \mathrm{P}=0.017 \\
{ }^{3} \mathrm{P}=0.644\end{array}$ & $\begin{array}{l}\mathrm{I} P=1.00 ;{ }^{2} \mathrm{P}=0.017 ;{ }^{3} \mathrm{P}=0.644 \\
{ }^{4} \mathrm{P}=1.00\end{array}$ \\
\hline RBCs $\left(x 10^{6} / \mathrm{ul}\right)$ & $7.34 \pm 0.45$ & $4.69 \pm 1.07$ & $5.91 \pm 1.65$ & $6.4 I \pm I .07$ & $7.25 \pm 0.40$ \\
\hline Significance & - & ${ }^{I} P=0.011$ & ${ }^{I} P=0.122 ;{ }^{2} P=0.181$ & $\begin{array}{l}{ }^{I} P=0.298 ;{ }^{2} P=0.070 \\
{ }^{3} P=0.568\end{array}$ & $\begin{array}{l}{ }^{1} P=0.924 ;{ }^{2} P=0.013 ;{ }^{3} P=0.143 ; \\
{ }^{4} P=0.34 I\end{array}$ \\
\hline HGB (g/dl) & $16.43 \pm 1.04$ & $7.73 \pm 1.37$ & $|2.87 \pm 0.3|$ & $13.77 \pm 1.70$ & $15.83 \pm 0.97$ \\
\hline Significance & - & IP $<0.0001$ & ${ }^{I} P=0.004 ;{ }^{2} P<0.000 I$ & $\begin{array}{l}{ }^{1} P=0.019 ; \\
{ }^{2} P<0.0001 ;{ }^{3} P=0.370\end{array}$ & $\begin{array}{l}{ }^{1} P=0.545 ;{ }^{2} P<0.000 I \\
{ }^{3} P=0.01 I ;{ }^{4} P=0.056\end{array}$ \\
\hline НCT (\%) & $44.77 \pm 3.78$ & $24.37 \pm 4.11$ & $38.27 \pm 0.93$ & $43.23 \pm 2.90$ & $54.20 \pm 3.34$ \\
\hline Significance & - & 'P $<0.000$ I & ${ }^{I} \mathrm{P}=0.033 ;{ }^{2} \mathrm{P}<0.000 \mathrm{I}$ & $\begin{array}{l}{ }^{I} P=0.572 ;{ }^{2} P<0.0001 ; \\
{ }^{3} P=0.010\end{array}$ & $\begin{array}{l}{ }^{I} P=0.872 ;{ }^{2} P<0.000 I \\
{ }^{3} P=0.025 ;{ }^{4} P=0.47 I\end{array}$ \\
\hline $\mathrm{MCV}(\mathrm{FL})$ & $56.50 \pm 0.10$ & $54.57 \pm 3.96$ & $55.43 \pm 1.46$ & $55.80 \pm 2.21$ & $55.63 \pm 6.75$ \\
\hline Significance & - & ${ }^{I} P=0.536$ & ${ }^{I} \mathrm{P}=0.731 ;{ }^{2} \mathrm{P}=0.780$ & $\begin{array}{l}{ }^{1} P=0.821 ;{ }^{2} P=0.691 ; \\
{ }^{3} P=0.906\end{array}$ & $\begin{array}{l}{ }^{1} \mathrm{P}=0.780 ;{ }^{2} \mathrm{P}=0.73 \mathrm{I} ;{ }^{3} \mathrm{P}=0.948 \\
{ }^{4} \mathrm{P}=0.957\end{array}$ \\
\hline $\mathrm{MCH}(\mathrm{pg})$ & $\mid 8.57 \pm 0.32$ & $18.33 \pm 0.12$ & $18.63 \pm 0.38$ & $|8.47 \pm 0.2|$ & $18.10 \pm 0.36$ \\
\hline Significance & - & ${ }^{\prime} P=0.355$ & ${ }^{I} \mathrm{P}=0.787 ;{ }^{2} \mathrm{P}=0.240$ & $\begin{array}{l}{ }^{I} P=0.686 ;{ }^{2} P=0.591 ; \\
{ }^{3} P=0.504\end{array}$ & $\begin{array}{l}{ }^{1} \mathrm{P}=0.081 ;{ }^{2} \mathrm{P}=0.355 ;{ }^{3} \mathrm{P}=0.05 \mathrm{I} ; \\
{ }^{4} \mathrm{P}=0.158\end{array}$ \\
\hline $\mathrm{MCHC}(\mathrm{g} / \mathrm{dl})$ & $34.37 \pm 1.29$ & $33.07 \pm 1.24$ & $33.63 \pm 0.46$ & $33.10 \pm 1.5 \mid$ & $27.27 \pm 11.84$ \\
\hline Significance & - & ${ }^{I} P=0.774$ & ${ }^{I} \mathrm{P}=0.87 \mathrm{I} ;{ }^{2} \mathrm{P}=0.900$ & $\begin{array}{l}{ }^{I} P=0.780 ;{ }^{2} P=0.994 ; \\
{ }^{3} P=0.906\end{array}$ & $\begin{array}{l}{ }^{1} P=0.139 ;{ }^{2} P=0.218 ;{ }^{3} P=0.180 ; \\
{ }^{4} P=0.215\end{array}$ \\
\hline RDW (\%) & $19.30 \pm 9.30$ & $15.20 \pm 0.87$ & $15.27 \pm 1.52$ & $|7.33 \pm| .3 \mid$ & $14.86 \pm 0.39$ \\
\hline Significance & - & ${ }^{I} P=0.267$ & ${ }^{I} \mathrm{P}=0.275 ;{ }^{2} \mathrm{P}=0.985$ & $\begin{array}{l}{ }^{1} P=0.586 ;{ }^{2} P=0.555 \\
{ }^{3} P=0.567\end{array}$ & $\begin{array}{l}{ }^{1} \mathrm{P}=0.232 ;{ }^{2} \mathrm{P}=0.924 ;{ }^{3} \mathrm{P}=0.909 \\
{ }^{4} \mathrm{P}=0.495\end{array}$ \\
\hline $\begin{array}{l}\text { Platelets }\left(\times 10^{3} /\right. \\
\text { ul) }\end{array}$ & $772.67 \pm 40.10$ & $742.67 \pm 28.68$ & $674.00 \pm 66.36$ & $702.33 \pm 82.11$ & $748.67 \pm 65.77$ \\
\hline Significance & - & ${ }^{I} P=0.553$ & ${ }^{I} \mathrm{P}=0.071 ;{ }^{2} \mathrm{P}=0.190$ & $\begin{array}{l}{ }^{I} P=0.181 ;{ }^{2} P=0.428 \\
{ }^{3} P=0.575\end{array}$ & $\begin{array}{l}{ }^{1} P=0.634 ;{ }^{2} P=0.905 ;{ }^{3} P=0.157 ; \\
{ }^{4} P=0.365\end{array}$ \\
\hline MPV (FL) & $6.40 \pm 0.00$ & $8.07 \pm 1.81$ & $6.50 \pm 0.66$ & $6.97 \pm 1.07$ & $6.43 \pm 0.40$ \\
\hline Significance & - & ${ }^{\prime} P=0.069$ & ${ }^{I} P=0.905 ;{ }^{2} P=0.085$ & $\begin{array}{l}{ }^{1} P=0.505 ;{ }^{2} P=0.209 \\
{ }^{3} P=0.581\end{array}$ & $\begin{array}{l}{ }^{\mathrm{P}} \mathrm{P}=0.968 ;{ }^{2} \mathrm{P}=0.074 ;{ }^{3} \mathrm{P}=0.937 ; \\
{ }^{4} \mathrm{P}=0.530\end{array}$ \\
\hline
\end{tabular}

'P, significance versus GI; ${ }^{2} \mathrm{P}$, significance versus G2; ${ }^{3}$, significance versus G3; ${ }^{4}$, significance versus G4; WBCs, white blood cells; RBCs, red blood cells; HGB, hemoglobin; HCT, hematocrit; MCV, mean corpuscular volume; $\mathrm{MCH}$, mean corpuscular hemoglobin; $\mathrm{MCHC}$, mean corpuscular hemoglobin concentration; RDW, red cell distribution width; MPV, mean platelet volume 
Table 2 Comparison of kidney functions between different studied groups

\begin{tabular}{|c|c|c|c|c|c|}
\hline Parameters & $\begin{array}{l}\text { GI } \\
\text { (Control) }\end{array}$ & G2 (acetaminophen) & $\begin{array}{l}\text { G3 } \\
\text { (AM } 200 \mathrm{mg} / \mathrm{kg})\end{array}$ & $\begin{array}{l}\text { G4 } \\
\text { (AM } 400 \mathrm{mg} / \mathrm{kg})\end{array}$ & $\begin{array}{l}\text { G5 } \\
\text { (AM } 600 \mathrm{mg} / \mathrm{kg})\end{array}$ \\
\hline Urea (mg/dl) & $33.7 I \pm 3.23$ & $153.17 \pm 30.99$ & $56.46 \pm 10.13$ & $41.88 \pm 2.10$ & $39.25 \pm 3.35$ \\
\hline Significance & - & ${ }^{I} P<0.000 \mid$ & ${ }^{I} P=0.088 ;{ }^{2} P<0.000 I$ & $\begin{array}{l}{ }^{I} P=0.513 ;{ }^{2} P<0.000 I ; \\
{ }^{3} P=0.254\end{array}$ & $\begin{array}{l}{ }^{1} \mathrm{P}=0.655 ;{ }^{2} \mathrm{P}<0.0001 ; \\
{ }^{3} \mathrm{P}=0.184 ;{ }^{4} \mathrm{P}=0.831\end{array}$ \\
\hline Creatinine $(\mathrm{mg} / \mathrm{dl})$ & $3.47 \pm 0.23$ & $6.27 \pm 0.46$ & $4.67 \pm 0.46$ & $4.13 \pm 0.23$ & $3.85 \pm 0.13$ \\
\hline Significance & - & $I P<0.000 \mid$ & ${ }^{I} \mathrm{P}=0.00 \mathrm{I} ;{ }^{2} \mathrm{P}<0.000 \mathrm{I}$ & $\begin{array}{l}{ }^{\mathrm{P}} \mathrm{P}=0.034 ;{ }^{2} \mathrm{P}<0.000 \mathrm{I} \\
{ }^{3} \mathrm{P}=0.077\end{array}$ & $\begin{array}{l}{ }^{I} P=0.188 ; \\
{ }^{2} P<0.0001 ;{ }^{3} P=0.013 ; \\
{ }^{4} P=0.320\end{array}$ \\
\hline
\end{tabular}

'P, significance versus GI; ${ }^{2} \mathrm{P}$, significance versus $\mathrm{G} 2 ;{ }^{3} \mathrm{P}$, significance versus $\mathrm{G} 3 ;{ }^{4} \mathrm{P}$, significance versus G4

Table 3 Comparison of oxidative stress markers between different studied groups

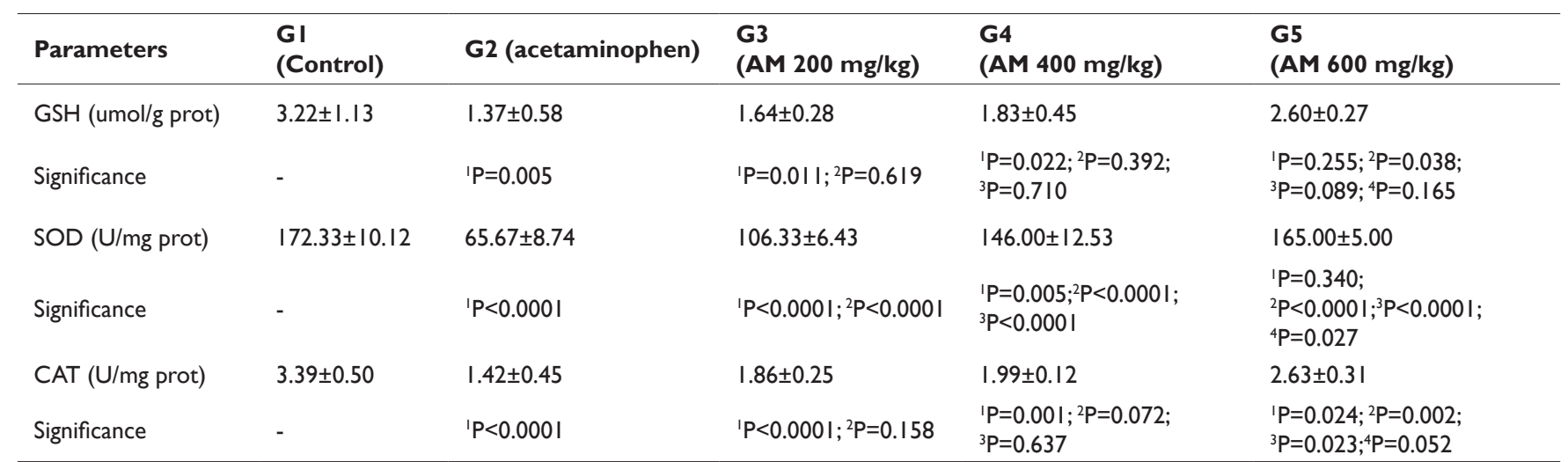

'P, significance versus GI; ${ }^{P}$, significance versus $\mathrm{G} 2 ;{ }^{3} \mathrm{P}$, significance versus $\mathrm{G} 3 ;{ }^{4} \mathrm{P}$, significance versus $\mathrm{G} 4$, Significance was made using $\mathrm{GSH}$, glutathione; SOD, superoxide dismutase; CAT, catalase
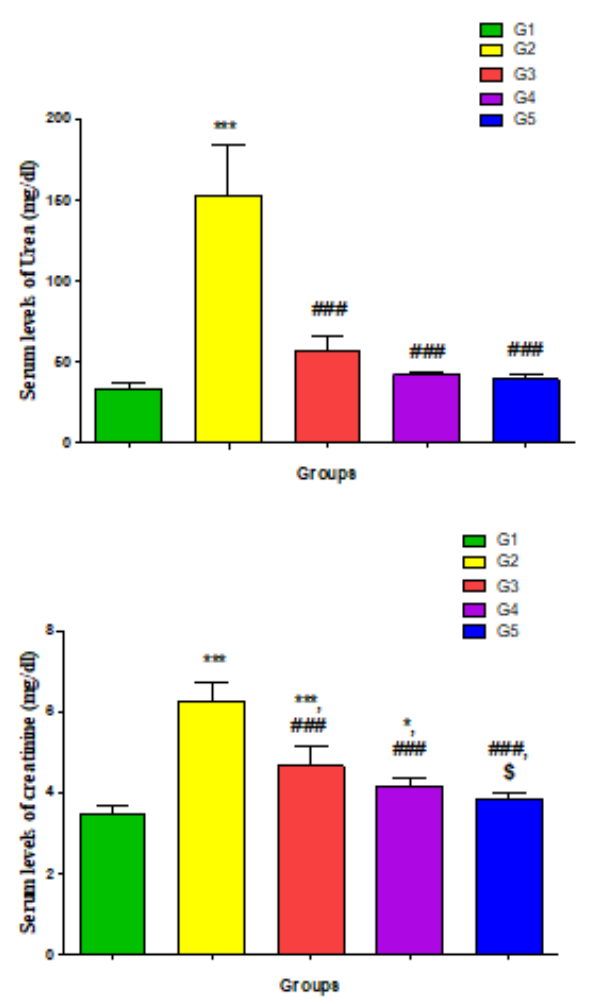

Figure I Comparison of urea and creatine serum levels in between different studied groups. ${ }^{*}$ : significance versus GI; ${ }^{*}$ : significance versus G2. \$: significance versus G3. *: $\mathrm{P}<0.05$; **: $\mathrm{P}<0.0$ I; **: $\mathrm{P}<0.00$ I.
Low power photographs of paraffin sections from mice kidney in different studied groups showed mild differences between cortical thickness of in G2, G3, G4 and G5 versus control group (Figure 3). Low power (x200) photographs of paraffin sections from mice kidney showing the histology of outer cortex showed deformity and capillary atrophy of renal corpuscles in G2: acetaminophen (2000 Mg/kg/b.w.) compared to normal corpuscles in G1: control and other treated groups (G3-G5). Renal tubules of G2 (acetaminophen) showed drastic changes in the form of dilation (wide lumina) due to degeneration of their lining epithelium with presence of desquamated cells in side their lumen compared to normal control Mild protection was observed in G3 (mild dose) but still dilated tubules with luminal desquamated cells, moderate with medium dose (G4) and marked protection in G5 (high dose) with kidney tissue nearly similar to control (Figure 4).

High power (X600) photographs of paraffin blocks from mice renal cortex stained by H\&E showed G1: control with normal renal corpuscle and glomerular capillaries. Normal renal tubules with narrow clear lumina and healthy epithelial lining. G2: acetaminophen toxicity $(2000 \mathrm{mg} / \mathrm{kg} / \mathrm{b}$.w.) showed marked deformity and atrophy of renal corpuscles and glomerular capillaries, renal tubules showed marked dilation, epithelial lining atrophy and cell debris and casts inside the lumina, G3: acetaminophen +LD of Alhagi maurorum roots $(200 \mathrm{mg} /$ $\mathrm{kg} / \mathrm{b}$.w.) showed mild protection of renal corpuscle and glomeruli. Renal tubules showed dilation and luminal casts and degenerated. G4: acetaminophen +MD Alhagi maurorum roots $(400 \mathrm{Mg} / \mathrm{kg} / \mathrm{b}$.w.) showed evident protection of kidney corpuscle glomerular capillaries and tubules but their cells had slightly inactive dark nuclei which nearly looked similar to control group. G5: acetaminophen +HD Alhagi maurorum roots $(600 \mathrm{Mg} / \mathrm{kg} /$ b.w.) revealed protection of renal corpuscle glomerular capillaries and tubules which showed active 
lightly stained nuclei which nearly looked similar to control group (Figure 5).
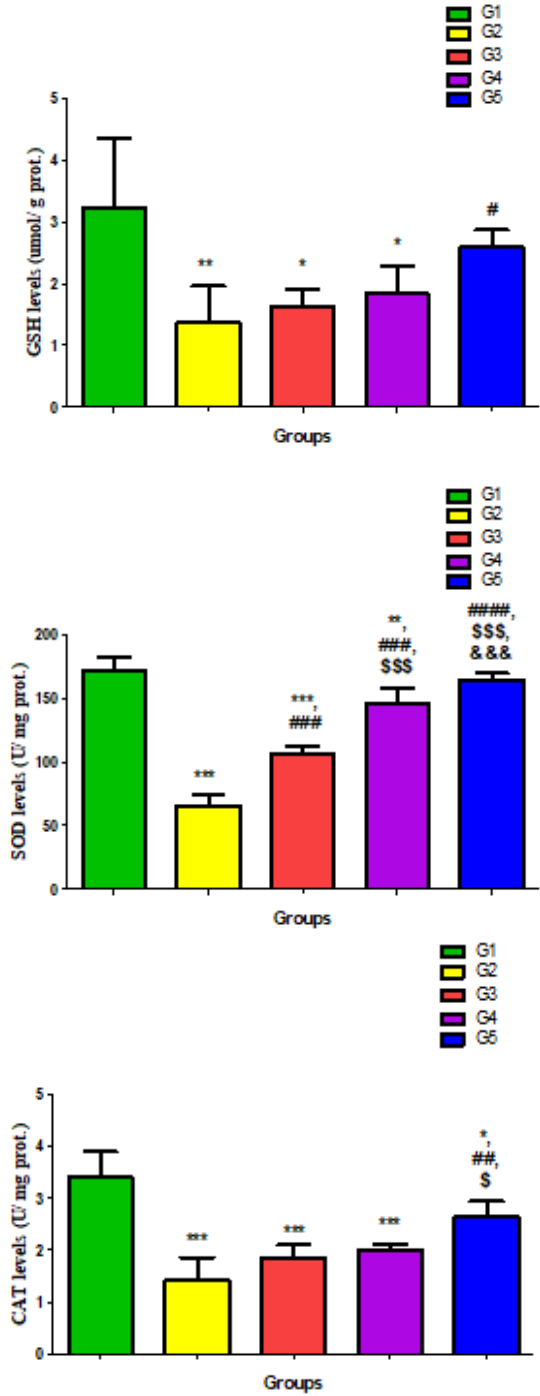

Figure 2 Comparison of glutathione (GSH), superoxide dismutase (SOD) and catalase (CAT) levels between different studied groups. "P: significance versus GI; \#: significance versus G2. \$: significance versus G3; \&: significance versus G4. *: $P<0.05 ; * *: P<0.01 ; * *: P<0.001$.

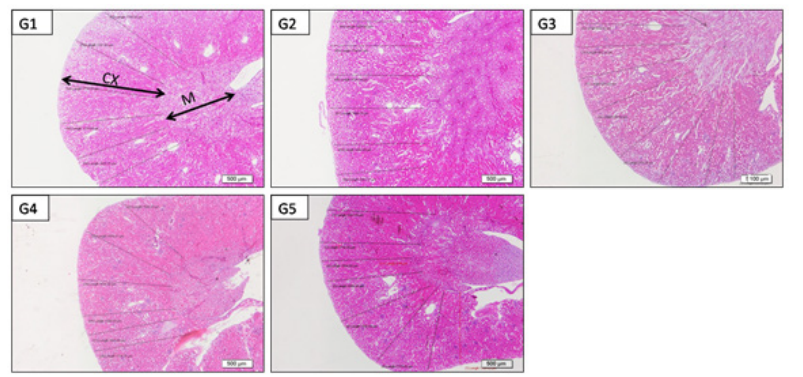

Figure 3 Low power photographs of paraffin sections from mice kidney (GI: control, G2: acetaminophen toxicity, G3: acetaminophen+LD $200 \mathrm{mg} /$ $\mathrm{kg}$ of Alhagi maurorum extract; G4: acetaminophen+MD $400 \mathrm{mg} / \mathrm{kg}$ of Alhagi maurorum extract and G5: acetaminophen+HD $600 \mathrm{mg} / \mathrm{kg}$ of Alhagi maurorum extract) showing an outer cortex (CX) and inner medulla (M), notice the mild differences between cortical thickness of different groups.

High power (X600) photographs of paraffin sections from mice kidney medulla stained by H\&E showed G1: control with normal renal tubules with narrow clear lumina and intact epithelial lining. G2: acetaminophen toxicity $(2000 \mathrm{mg} / \mathrm{kg} /$ b.w.) showed renal tubules with marked dilation, epithelial lining atrophy and cell debris and casts inside the lumina; G3: acetaminophen $+\mathrm{LD}$ of Alhagi maurorum roots $(200 \mathrm{mg} / \mathrm{kg} / \mathrm{b}$.w.) showed mild protection. Renal tubules still showed dilation and luminal casts and degenerated cells. G4: acetaminophen +MD Alhagi maurorum roots $(400 \mathrm{mg} / \mathrm{kg} / \mathrm{b}$.w.) showed protection of renal tubules but their cells have slightly inactive dark nuclei which nearly looked similar to control group. G5: acetaminophen +HD Alhagi maurorum roots $(600 \mathrm{mg} / \mathrm{kg} / \mathrm{b}$.w. $)$ marked protection of renal tubules that here showed active lightly stained nuclei and nearly looked similar to control group (Figure 6).

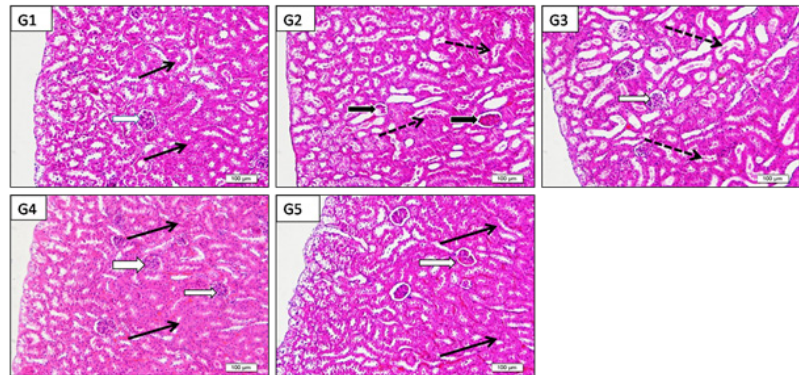

Figure 4 Low power $(\times 200)$ photographs of paraffin sections from mice kidney (GI:control, G2:acetaminophen toxicity, G3: acetaminophen+LD 200 $\mathrm{mg} / \mathrm{kg}$ of Alhagi maurorum extract; G4:acetaminophen +MD $400 \mathrm{mg} / \mathrm{kg}$ of Alhagi maurorum extract and G5: acetaminophen +HD $600 \mathrm{mg} / \mathrm{kg}$ of Alhagi maurorum extract) showing the histology of outer cortex notice the deformity and capillary atrophy of renal corpuscles (black arrows) in G2:acetaminophen (2000 $\mathrm{Mg} / \mathrm{kg} / \mathrm{b} . w$. .) compared to normal corpuscles (white arrows) in GI: control and other treated groups (G3-G5). Renal tubules of G2 (acetaminophen) showed drastic changes (dotted arrows) in the form of dilation (wide lumina) due to degeneration of their lining epithelium with presence of desquamated cells in side their lumen compared to normal control (black arrows). Notice, mild protection was observed in G3: low dose but still dilated tubules with luminal desquamated cells, moderate with medium dose (G4) and marked protection in G5 (high dose) with kidney tissue nearly similar to GI: control.

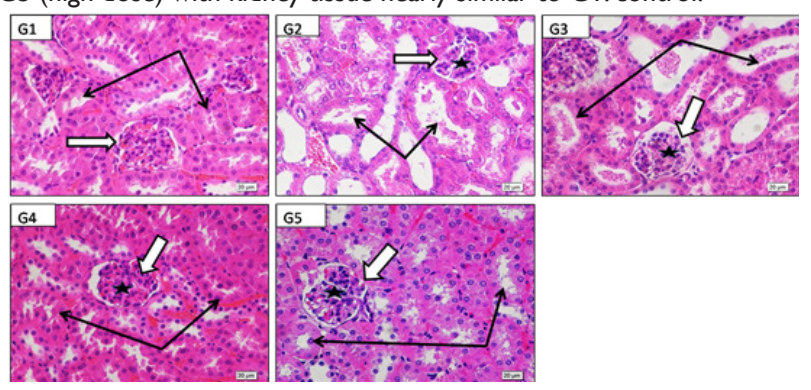

Figure 5 High power (X600) photographs of paraffin blocks from mice renal cortex stained by H\&E to show G: control with healthy renal corpuscle (white arrow) and its glomerular capillaries (star). Normal renal tubules with narrow clear lumina and healthy epithelial lining (black arrows). G2: acetaminophen toxicity $(2000 \mathrm{Mg} / \mathrm{kg} / \mathrm{bw})$ showing marked deformity and atrophy of renal corpuscles (white arrow) and glomerular capillaries (star), renal tubules showed marked dilation, epithelial lining atrophy and cell debries and casts inside the lumina (black arrows), G3: acetaminophen+LD of Alhagi maurorum roots $(200 \mathrm{Mg} / \mathrm{kg} / \mathrm{b}$.w.) showing mild protection of renal corpuscle (white arrow) and glomeruli (star) .Renal tubules still showed dilation and luminal casts and degenerated cells (black arrows). G4:acetaminophen+MD Alhagi maurorum roots $(400 \mathrm{Mg} / \mathrm{kg} / \mathrm{b}$.w.) showing evident protection of kidney corpuscle (white arrow), glomerular capillaries (star) and kidney tubules (black arrows) but their cells have slightly inactive dark nuclei which nearly looked similar to control group. G5: acetaminophen+HD Alhagi maurorum roots $(600 \mathrm{Mg} / \mathrm{kg} / \mathrm{b}$.w.) showing also marked protection of both kidney corpuscle (white arrow), glomerular capillaries (star) and kidney tubules (black arrows) which here showed active lightly stained nuclei which nearly looked similar to control group. 


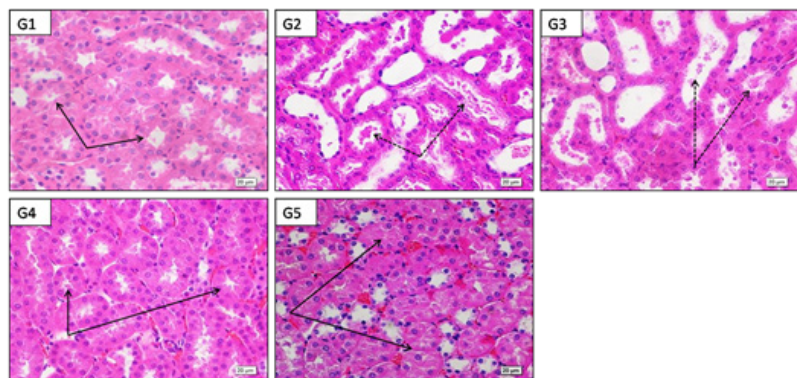

Figure 6 High power $(X 600)$ photographs of paraffin sections from mice kidney medulla stained by H\&E to show GI: control with normal. Normal renal tubules with narrow clear lumina and healthy epithelial lining (black arrows) .G2: acetaminophen toxicity $(2000 \mathrm{mg} / \mathrm{kg} / \mathrm{b} . w$.) showing renal tubules with marked dilation, epithelial lining atrophy and cell debris and casts inside the lumina (black arrows); G3: acetaminophen+LD of Alhagi maurorum roots (200 $\mathrm{mg} / \mathrm{kg} / \mathrm{b}$.w.) showing mild protection . Renal tubules still showed dilation and luminal casts and degenerated cells (black arrows). G4: acetaminophen+MD Alhagi maurorum roots $(400 \mathrm{mg} / \mathrm{kg} / \mathrm{b} . \mathrm{w}$.) showing protection of renal tubules (black arrows) but their cells have slightly inactive dark nuclei which nearly looked similar to control group. G5: acetaminophen+HD Alhagi maurorum roots $(600 \mathrm{mg} / \mathrm{kg} / \mathrm{b} . w$.) markedprotection of renal tubules (black arrows) which here showed active lightly stained nuclei and nearly looked similar to control group.

\section{Discussion}

Acetaminophen increased WBCs and its differential can be explained by induction acute kidney injury that led to inflammation. Meanwhile, reduction of RBCs, hemoglobin and hematocrit may be explained by the suppression effects of acetaminophen on erythropoietin secreted by the kidney. ${ }^{13}$. These changes improved after Alhagi maurorum roots extract oral administration due to improvement of the kidney functions and structure. Acute acetaminophen toxicity is one of the most reasons of renal damages ${ }^{14}$ Renal destructionintensity by acetaminophen depends upon cytochrome p450 system and glutathione deposits. In kidneys, cytochrome p450 is chiefly present in proximal convoluted tubule and only little amount in glomerulus, distal convoluted tubules and collecting ducts. On the other hand, because absorption and secretion actionss in proximal convoluted tubules, toxic metabolites concentrations are more in tubules than other renal parts. ${ }^{14,15}$

The mechanism of acetaminophen-maderenal toxicity and hepatic toxicity reported by other researches and revealed that acetaminophen led to oxidative stress and kidneydestruction when utilized at high doses. ${ }^{16-19}$ It was concluded that acetaminophen intakeled to significant elevation in the blood urea nitrogen, uric acid and creatinine values, indicating renal destruction associated with degeneration of tubular epithelium and infiltration of inflammatory cells in kidneys. ${ }^{20-22}$ Histological results consistent with biochemical results. Furthermore, histopathology of renal' findings emphasis the protective actions of Alhagi maurorum root ethanol extract versus acetaminophen-induced kidney destructions as it is revealed by reducing of kidney destruction as infiltration of inflammatory cells, congestion, edema, degeneration and necrosis mainly at dose of $600 \mathrm{mg} / \mathrm{kg}$ of Alhagi maurorumethanol root extract. Nonetheless, effect of Alhagi maurorum root extract at the dose of $200 \mathrm{mg} / \mathrm{kg}$ and $400 \mathrm{mg} / \mathrm{kg}$, mild necrosis, degeneration, edema was found. This might be due to anti-oxidant action of Alhagi maurorum extract.

Histological results are also in line with biochemical results. Furthermore, histopathology of kidneys' results emphasis the protective actions of Alhagi maurorum root ethanol extract versus acetaminophen-induced kidney destruction as it is shown by decreased of renal destruction as inflammatory cell infiltrations, congestion, edema, degeneration and necrosis mainly at dose of $600 \mathrm{mg} / \mathrm{kg}$ of Alhagi maurorum ethanol root extract. Nonetheless, action of Alhagi maurorum root extract at dose of $200 \mathrm{mg} / \mathrm{kg}$ and $400 \mathrm{mg} / \mathrm{kg}$, mild necrosis, degeneration, edema was found. This might be due to antioxidant action of Alhagi maurorum extract.

\section{Conclusion}

From the results obtain by this research, it can be concluded that Alhagi maurorum ethanol root extract especially high dose $(600 \mathrm{mg} / \mathrm{kg})$ had protected effects against renal damage induced by acetaminophen. That may be due to its anti-oxidant activity. So, this study recommended oral administration of Alhagi maurorum to treated renal damage induced by acetaminophen intake. Finally, the medical usage of this herb can be promising and further studies are required to determine the active ingredients of this plant to establish a new herbal drug to treated kidney's failure patients.

\section{Acknowledgments}

None.

\section{Conflicts of interest}

No potential conflict of interest was reported by the authors.

\section{Funding}

The author declares there are no conflicts of interest.

\section{References}

1. Kharchoufa L, Merrouni IA, Yamani A, et al. Profile on medicinal plants used by the people of North Eastern Morocco: toxicity concerns. Toxicon. 2018;154:90-113

2. Kuete V. Toxicological survey of African medicinal plants: Elsevier. 2014.

3. Atta AH, Mouneir S M. Antidiarrhoeal activity of some Egyptian medicinal plant extracts. Journal of Ethnopharmacology. 2004;92(23):303-309

4. Shakiba Y, Rezatofighi SE, Nejad SMS, et al. Antiviral Activity of Alhagi maurorum Medik's Methanolic Extract on Foot and Mouth Disease Virus (FMDV) in Cell Cultures. Jundishapur J Nat Pharm Prod. 2016;11(3):1-5.

5. Marashdah M, Al-Hazimi H. Pharmacological activity of ethanolic extract of Alhagi maurorum roots. Arabian Journal of Chemistry. 2010;3(1):39-42.

6. Gargoum HM, Muftah SS, Al Shalmani S, et al. Phytochemical screening and investigation of the effect of Alhagi maurorum (camel thorn) on carbon tetrachloride, acetaminophen and adriamycin induced toxicity in experimental animals. Journal of Scientific and Innovative Research. 2013;2(6):1023-1033.

7. Loizzo MR, Rashed K, Sai A, et al. Antiproliferative and antioxidant properties of Alhagi maurorum Boiss (Leguminosae) aerial parts. Industrial Crops and Products. 2014;53:289-295.

8. Wang ZK, Liu ZY, Yu HB. Protective effect of telmisartan on rats with renal failure and its mechanism. Asian Pacific Journal of Tropical Medicine. 2015;8(6):498-501.

9. Li QM, Luo JP, Pan LH, et al. Evaluation of renoprotective effect of Chinese chive extracts on adenine-induced chronic renal failure. Food Science and Human Wellness. 2018;7(4): 260-265. 
10. Awaad AS, El-Meligy R, Qenawy S, et al. Anti-inflammatory, antinociceptive and antipyretic effects of some desert plants. Journal of Saudi Chemical Society. 2011;15(4):367-373.

11. Shaker E, Mahmoud H, Mnaa S. Anti-inflammatory and anti-ulcer activity of the extract from Alhagi maurorum (camelthorn). Food and Chemical Toxicology. 2010;48(10):2785-2790.

12. Roy S, Pradhan S, Das K, et al. Acetaminophen induced kidney failure in rats: a dose response study. Journal of Biological Sciences. $215 ; 15(4): 87$

13. Oyedeji K, Bolarinwa A, Ojeniran S.. Effect of paracetamol (acetaminophen) on haematological and reproductive parameters in male albino rats. IOSR Journal of Pharmacy and Biological Sciences (IOSR-JPBS). 2013;4(6):1-6.

14. Mazer M, Perrone J. Acetaminophen-induced nephrotoxicity: pathophysiology, clinical manifestations, and management. Journal of Medical Toxicology. 2008;4(1):2-6.

15. McGill MR, Williams CD, Xie Y, et al. Acetaminophen-induced liver injury in rats and mice: comparison of protein adducts, mitochondrial dysfunction, and oxidative stress in the mechanism of toxicity. Toxicology and applied pharmacology. 2012;264(3):387-394.

16. Honmore V, Kandhare A, Zanwar AA, et al. Artemisia pallens alleviates acetaminophen induced toxicity via modulation of endogenous biomarkers. Pharmaceutical biology. 2015;53(4):571-581.
17. Moshaie-Nezhad P, Iman M, Faed Maleki F, et al. Hepatoprotective effect of Descurainia sophia seed extract against paracetamol-induced oxidative stress and hepatic damage in mice. $J$ Herbmed Pharmacol. 2018;7(4)

18. Naguib YM, Azmy RM, Samaka RM, et al. Pleurotus ostreatus opposes mitochondrial dysfunction and oxidative stress in acetaminopheninduced hepato-renal injury. BMC Complementary and Alternative Medicine. 2014;14(1):1-12.

19. Olaleye MT, Rocha BJ. Acetaminophen-induced liver damage in mice: effects of some medicinal plants on the oxidative defense system. Experimental and Toxicologic Pathology. 2008;59(5):319-327.

20. Das J, Ghosh J, Manna P, et al. Taurine protects acetaminophen-induced oxidative damage in mice kidney through APAP urinary excretion and CYP2E1 inactivation. Toxicology. 2010;269(1):24-34.

21. Kavak S, Pirincci N, Demir H, et al. The Prolidase Activity, Oxidative Stress, and Nitric Oxide Levels of Bladder Tissues with or Without Tumor in Patients with Bladder Cancer. 2017.

22. Marashdah MS. New natural compound for the enlargement of the ureter. Arabian Journal of Chemistry. 2014;7(3):381-383. 